
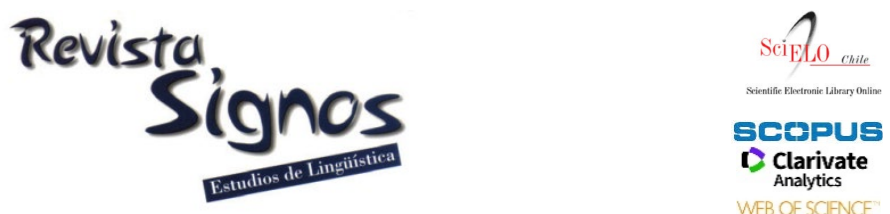

WEB OF SCIENCE

\title{
Relaciones semántico-cognitivas de los neologismos formados con los sufijos -ismo e - ista en la variante del español de Colombia
}

\section{Semantic and cognitive relations of neologisms with -ismo and- ista suffixes in the colombian spanish variant}

\section{Óscar Andrés Calvache Dulce \\ UnivERSIDAD AUtónOMA DE MANIZALES COLOMBIA \\ oscarac@autonoma.edu.co}

\author{
Mercedes Suárez \\ de la Torre \\ UNIVERSIDAD AUTÓNOMA DE MANIZALES \\ COLOMBIA \\ mercedessuarez@autonoma.edu.co
}

Andrea Suárez

Salazar

UNIVERSIDAD AUTÓNOMA DE MANIZALES COLOMBIA andreasuarez@autonoma.edu.co

Recibido: 07-XII-2017 / Aceptado: 22-V-2019

DOI: $10.4067 /$ S0718-09342020000200369

\section{Resumen}

Los eventos históricos, las tendencias económicas, culturales, filosóficas y artísticas propias de un grupo de hablantes constituyen factores contextuales importantes que influyen en la creación léxica de las lenguas, entre ellas el español en su variante colombiana. En el marco del Proyecto Internacional Antenas Neológicas adscrito al Observatorio de Neología de la Universidad Pompeu Fabra de Barcelona, el trabajo realizado por la Antena Neológica Colombiana (ANECOL) evidenció que uno de los procesos más productivos de formación de palabras en el contexto colombiano es la sufijación. Por consiguiente, en este artículo se analizaron los neologismos formados por los sufijos -ismo e -ista desde una perspectiva semántico-cognitiva. La Teoría de la Semántica de los Marcos (Fillmore, 1975, 1977, 1982, 1985) y los Modelos Cognitivos Idealizados (MCI) (Lakoff, 1987) constituyeron el referente teórico. Para ello, se identificaron los neologismos formados por los sufijos -ismo e -ista que aparecían en el periódico El Tiempo de Colombia durante los años 2009-2010, procesados de manera semiautomática con la herramienta BUSCANEO. El análisis de la relación semántica dual y no dual (Muñoz, 2010) de los neologismos permitió establecer una clasificación inicial, la cual dio pie para un posterior análisis de los marcos, los MCI y los efectos prototípicos simples y complejos. Los resultados obtenidos mostraron que los neologismos en -ismo, por su carácter nominal, presentaron una estructura semánticocognitiva abstracta y convergieron en un mismo MCI. Los neologismos formados con el sufijo -ista con carácter adjetival, presentaron una estructura semántico-cognitiva concreta y se distanciaron del MCI. 
Palabras Clave: Relaciones semántico-cognitivas, neología, sufijación, modelos cognitivos idealizados, efectos prototípicos.

\begin{abstract}
Historical events, as well as economic, cultural, philosophical and artistic trends characteristic of a group of speakers are important contextual factors influencing the lexical creation of languages, including Spanish in its Colombian variant. Under the International Neological Antennas Project attached to the Observatory of Neology of the University Pompeu Fabra of Barcelona, the work done by the Colombian Neological Antenna (ANECOL) showed that one of the most productive processes of word formation within the Colombian context is suffixation. Accordingly, this article presents the analysis of neologisms formed by -ismo and -ista suffixes from a cognitive semantics perspective. The theory of frame semantics (Fillmore, 1975, 1977, 1982, 1985) and Idealized Cognitive Models (ICM) (Lakoff, 1987) constituted the theoretical framework. To do this, the neologisms formed by suffixes -ismo and -ista were identified in the Colombian newspaper El Tiempo, during the years 2009-2010, and processed semi-automatically with the BUSCANEO tool. Analysis of dual and nondual semantic relations (Muñoz, 2010) of neologisms allowed the researchers to establish an initial classification. This gave rise to further analysis of frames, ICM and the simple and complex prototypical effects. The results showed that the neologisms with -ismo, presented an abstract cognitive semantic structure and converged on the same ICM due to their nominal character. Neologisms formed with the suffix -ista had a particular cognitive semantic structure and diverged from the ICM when having an adjectival character.
\end{abstract}

Key Words: Semantic and cognitive relations, neology, suffixation, idealized cognitive models, prototype effects.

\title{
INTRODUCCIÓN
}

Uno de los procesos más productivos de formación de palabras nuevas (neologismos) en el contexto colombiano es la sufijación. En este estudio en particular, hemos tomado como base las formas léxicas -ismo e -ista para abordarlas desde una perspectiva semántico-cognitiva; esto es, concebir la semántica desde el punto de vista experiencial, con el fin de comprender que, de alguna manera, el 'significado se negocia' debido a factores no solo lingüísticos, sino también contextuales.

Tradicionalmente se han realizado estudios que buscan la comprensión de las palabras formadas por sufijación desde una perspectiva semántica (Cabré, Bayà, Bernal, Freixa, Solé \& Vallès, 2002) y se han descrito múltiples categorías con referencia a los sufijos estudiados (Cabré \& Rigau, 1986; Lang, 1992; Beniers, 1992; Lacuesta \& Bustos, 1999; Capanaga, 1999, 2001; Meyer-Lübke, 1890-1906; Alemany, 1920). Según Ibarretxe-Antuñano y Valenzuela (2012), una visión tradicionalmente aceptada asume que las palabras específicas corresponden a conceptos particulares, esencialmente idénticos en la mente de los hablantes, siendo así caracterizados en términos objetivos con base en el establecimiento de listas de trabajos semánticos, 
comprendidas en general por los seres humanos en un tiempo y contexto determinados. Contrario a esta postura, los planteamientos de la semántica cognitiva, teoría empírica susceptible de verificación, sostiene que el léxico nuevo en los hablantes está relacionado con modelos cognitivos que no solo se representan en términos de condiciones necesarias y suficientes, sino que permiten que dichos hablantes construyan significados y configuren interpretaciones en el marco de contextos que se activan en función de sus conocimientos y experiencias.

Así pues, el objetivo principal de este trabajo es describir, semántica y cognitivamente, las relaciones que conllevan a nuevos conceptos generales a partir del análisis de los contextos de uso en la prensa escrita de los neologismos objeto de estudio. En este sentido, las contribuciones de este estudio se extienden también a los dominios de especialidad, en tanto permite establecer de manera natural relaciones de tipo conceptual, necesarias hoy en día para el desarrollo de aplicaciones tecnológicas.

Para ello, en primer lugar, partimos de un corpus textual constituido por 72 ejemplares en línea entre los años 2009-2010 del periódico El Tiempo de circulación nacional. En segundo lugar, constituimos un corpus de análisis en dos fases y clasificamos las nuevas palabras formadas por sufijación de manera tal que pudiesen ser analizadas desde la Teoría de la Semántica de los Marcos y los Modelos Cognitivos Idealizados (MCI) (Fillmore, 1975, 1977, 1982, 1985; Lakoff, 1983, 1987). De este análisis deriva la posibilidad de observar si realmente los neologismos formados por los sufijos -ismo e -ista permiten elaborar relaciones de tipo concreto o abstracto y si dichas relaciones conducen a nuevas realidades o simplemente permanecen las ya existentes.

El artículo presenta una revisión teórica sobre los aportes que se han hecho de los sufijos -ismo e -ista, desde la perspectiva semántica. Posteriormente, se aborda la Teoría de la Semántica de los Marcos y de los MCI. Una vez desarrollada esta postura teórica, se detalla el procedimiento para conformar el corpus de análisis y se organizan los neologismos en dos categorías según su relación dual y no dual, componente que permite profundizar en el análisis. Además de describir estos dos tipos de relaciones, los resultados se exponen con base en los MCI y los efectos prototípicos simples y complejos. Por último, las conclusiones permiten corroborar el objetivo planteado.

\section{Marco teórico}

Con el fin de identificar y analizar los MCI (Lakoff, 1983, 1987) de los neologismos formados con los sufijos -ismo e -ista en la variante del español de Colombia, fue pertinente hacer una revisión teórica sobre los diversos aportes que se han hecho de estos sufijos solamente desde la perspectiva semántica. En este sentido, Capanaga (2001) afirma que -ismo e -ista se distinguen por su capacidad neológica y por el 
sensible y generalizado aumento de su frecuencia en los registros lexicográficos a partir de 1989, hecho que también observamos en la variante del español de Colombia. La sufijación, en palabras de Santana, Carrera, Pérez y Rodríguez (2005: 1):

“...constituye el procedimiento de formación de palabras más importante en español. Entre la palabra original y el sufijo se desarrolla una interacción dinámica en los ámbitos semántico, funcional y formal que da lugar a una nueva palabra vinculada con la original".

\subsection{Desde la perspectiva semántica}

\subsubsection{Sufijo -ismo}

El estudio realizado por Cabré y Rigau (1986) afirma que el sufijo -ismo es un recurso de creación de palabras nuevas cuya categoría gramatical es la de sustantivos. Estos, según los autores, generalmente hacen referencia a nombres, originariamente abstractos, que designan movimiento o doctrina. Unos años más tarde, Lang (1992) y Beniers (1992) retomaron la anterior definición y adicionaron un matiz semántico importante que tuvo diversas implicaciones en estudios posteriores: el sufijo -ismo contribuye en la denominación de movimientos políticos reconocidos o tendencias más informales de pensamiento sociopolítico y cultural (ej. fascismo - marxismo).

Posteriormente, Lacuesta y Bustos (1999) establecieron una clasificación semántica de las diferentes palabras formadas con el sufijo -ismo, tomando como punto de partida los postulados presentados anteriormente (Cabré \& Rigau, 1986; Lang, 1992; Beniers, 1992). Esta clasificación constituye un aporte a la comprensión del sufijo ismo, a su contribución semántica cuando aparece acompañado de diversos tipos de radicales y a su relación con distintas áreas de conocimiento. A continuación se explican las cuatro acepciones que comprenden dicha clasificación:

a) Grupo que expresa opiniones de diferentes áreas: Lacuesta y Bustos (1999) afirman que el sufijo -ismo puede no solo referirse a la política o a la cultura, como lo afirmaron en su momento Lang (1992) y Beniers (1992), sino también a otros aspectos de la vida y de las sociedades: desde la política, encontramos palabras como 'comunismo' y 'fascismo'; desde las ciencias económicas encontramos las palabras ‘capitalismo' y ‘proteccionismo' y desde las creencias religiosas u orientaciones filosóficas, podrían mencionarse las palabras ‘budismo' y 'kantismo'. Asimismo, 'darwinismo' y 'generativismo' se refieren a ciencias biológicas o históricas y 'romanticismo' y ‘cubismo', a tendencias artísticas.

b) Grupo que designa caracteres, cualidades o formas de organización social o política: en esta categoría se ubican las palabras asociadas con las opiniones del grupo anterior, al igual que con sus consecuencias sociales. 'Autoritarismo', 'feminismo', ‘machismo' y 'caciquismo' son ejemplos de este grupo, pues se refieren a circunstancias históricas que derivan de eventos políticos específicos 
o del auge de ciertas corrientes económicas, religiosas, filosóficas, científicas o artísticas populares en determinada época y lugar.

c) Grupo que indica una actividad profesional: en este grupo podríamos mencionar las palabras 'ensayismo', 'automovilismo' y 'alpinismo'. Estas se refieren a disciplinas literarias y deportivas que constituyen la profesión u oficio de un grupo de personas. Es decir, algunas de ellas están enmarcadas en una actividad literaria o deportiva 'profesional', entendida como el oficio o el trabajo específico al cual se dedica una persona de tiempo completo. Esta claridad es importante puesto que tendrá implicaciones en el análisis del trabajo tomando como base esta postura.

d) Grupo que designa derivados propios de una jerga lingüística: en este grupo se ubican las palabras relacionadas con procesos lingüísticos o con sus resultados; es decir, con las dinámicas propias de las lenguas cuando entran y se transforman dentro de múltiples contextos geográficos del mundo. El ‘anglicismo', el 'laísmo’ y el 'leísmo’ tendrían relación con los procesos y el 'argentinismo', el 'americanismo' y el 'dequeísmo', con sus resultados o consecuencias (Lacuesta \& Bustos, 1999).

Por otra parte, Capanaga (1999) adiciona más acepciones a la anterior clasificación semántica del sufijo -ismo propuesta por Lacuesta y Bustos (1999), a partir del análisis que realiza sobre las formaciones neológicas en español (1989-1999). En este análisis adiciona tres nuevos valores semánticos a este sufijo:

e) Grupo que indica prácticas deportivas y actividades lúdicas: la autora afirma que -ismo ayuda a nombrar también deportes o juegos. Este es el caso de las palabras 'barranquismo', 'senderismo', 'atletismo' y ‘campismo'. En este sentido, y contrastando este nuevo valor semántico al previamente descrito sobre el grupo que indica una actividad profesional, aclaramos que se entiende como una actividad lúdica; es decir, una actividad de esparcimiento que no implica una dedicación de tiempo completo. Estas dos distinciones (profesional y lúdica), relacionadas con el área del deporte, se tendrán en cuenta en la presentación de los resultados del trabajo.

f) Grupo que hace valoraciones negativas: algunas palabras formadas por -ismo, normalmente en el campo de las actitudes, pueden tener una valoración negativa. Las palabras 'amiguismo', 'seguidismo' y 'colaboracionismo', por ejemplo, tienen esta connotación negativa y pudieron haber sido formadas en principio en el léxico político-social. Con el tiempo, esta valoración negativa pudo haberse extendido al léxico del resto de los ámbitos sociales, actitudes y comportamientos humanos.

g) Grupo que se refiere a la ciencia y tecnología: Capanaga (1999) también hace alusión al sufijo -ismo en las ciencias biológicas y tecnológicas. En cuanto a las ciencias biológicas, por ejemplo, hace referencia a palabras relacionadas con el 
campo de la medicina y, más concretamente, con aquellas que designan enfermedades, tales como: 'astigmatismo' e 'hipotiroidismo'.

\subsection{Sufijo -ista}

En la lengua griega, el sufijo -ista tenía un significado específico que se ha ido ampliando a través del tiempo. Este sufijo se utilizaba para hacer referencia únicamente a las diferentes tareas u oficios de las personas, pero actualmente se entiende con múltiples acepciones. El teórico Meyer-Lübke (1890-1906), por ejemplo, afirma que el sufijo -ista designa a las personas que poseen actitudes o estudios relacionados con una esfera culta; es decir, que va más allá de ejercer un simple oficio -como en el mundo griego-. Una profesión especializada que requiera una formación académica previa, según el autor, suele formarse con el sufijo -ista. Para tal caso, las palabras 'economista' y 'periodista' son ejemplos que ilustran la calidad y el tiempo de entrenamiento en el campo de la economía o el periodismo.

Además de esta primera acepción, se le atribuye al sufijo -ista el designar a los partidarios de las doctrinas filosóficas y de tendencias científicas o políticas. Este significado es más o menos equivalente al fenómeno semántico ocurrido con el sufijo -ismo y algunos ejemplos de esta acepción pueden ser las palabras 'terrorista' (tendencia política), 'humanista' (doctrina filosófica) y 'conductista' (tendencia científica).

Posteriormente, Alemany (1920) planteó en su obra, en concordancia con lo ya propuesto por Meyer-Lübke (1890-1906), que los términos en -ista denotan profesión, oficio, hábito u ocupación.

Lacuesta y Bustos (1999), por su parte, coincidieron con los autores anteriores en varios aspectos, pero desarrollaron otra clasificación semántica adicionando dos nuevas acepciones. En resumen, su propuesta con respecto a los derivados en -ista la expresaron en cuatro grandes categorías:

a) Designaciones profesionales: aunque coinciden con Meyer-Lübke (1890-1906), y Alemany (1920) en cuanto a las profesiones, su aporte más significativo consistió en la clasificación de las mismas. Ellos propusieron que el sufijo -ista puede aparecer en diversos campos del conocimiento: en el ámbito de la música, pueden mencionarse 'organista', 'solista' y 'sonidista'; en el campo de la literatura y la prensa, 'novelista' y 'cuentista'; y en el campo de la ciencia formal, 'analista' y 'economista'. Sucede lo mismo en las disciplinas financieras con las palabras 'accionista' y 'agiotista' y en el ámbito de los trabajos manuales con las palabras 'ceramista' y 'electricista'. Además de todas las anteriores, este sufijo puede denotar oficios marginales como 'perista' y 'espadista' y profesiones relacionadas con el mundo del espectáculo y del deporte como 'transformista' y 'equilibrista'. 
b) Convicciones políticas: este grupo se enmarca en una clasificación de diversos tipos de convicción. En consonancia con la definición de Meyer-Lübke (18901906) a este respecto, las palabras 'abolicionista' y ‘centrista' están relacionadas con el ámbito político; 'budista' y 'existencialista' con las creencias religiosas o filosóficas, 'estructuralista' con el campo de la ciencia y 'dadaísta' y 'expresionista', con el arte.

c) Descripciones del carácter de una persona, a veces con una valoración negativa: al referirse a la función negativa del sufijo -ismo, le atribuyeron al sufijo -ista actitudes humanas negativas, con los siguientes ejemplos: 'alarmista', 'camorrista' y 'cuentista'.

d) Designaciones de participantes o miembros de un grupo: las palabras 'asambleísta', y 'congresista' se utilizan para nombrar personas que hacen parte de un grupo reconocido socialmente.

Cabe mencionar que además de las acepciones anteriores, Capanaga (1999) otorgó un valor semántico adicional al sufijo -ista en su investigación. Este valor semántico se refiere al grupo de palabras que designan a los partidarios de un club deportivo, como 'barcelonista' y 'zaragocista'.

A manera de conclusión desde esta perspectiva lingüística, queremos mostrar que, si bien existen diversas clasificaciones de los valores semánticos de los sufijos -ismo e -ista, los contextos de uso de esos neologismos y el estudio de los MCI que subyacen a esos contextos, hacen que se cuestionen y se complementen las clasificaciones presentadas por los autores. Lo anterior se verá con mayor claridad en la presentación del análisis y de los resultados.

\subsection{Desde la perspectiva semántico-cognitiva}

Desde esta perspectiva, se tomó como punto de reflexión y análisis el concepto de Modelo Cognitivo Idealizado (MCI). Para ello, fue necesario referirse a la Teoría de la Semántica de los Marcos propuesta por Fillmore (1975, 1977, 1982, 1985), que postula que el conocimiento del significado de los elementos lingüísticos depende de las estructuras conceptuales subyacentes en las cuales se enmarca.

Según lo propuesto por Fillmore, el término 'marco' se entiende como el que nombra un sistema estructurado de conocimiento, que se guarda en la memoria a corto plazo y que se organiza para esquematizar la experiencia. Este autor argumenta que el significado de las palabras está subordinado a los marcos; por ello, la comprensión e interpretación del significado de cada palabra o de un conjunto de palabras, requieren un acceso a las estructuras de conocimiento que relacionan elementos y entidades asociadas a las diferentes escenas de la experiencia humana, considerándose así bases físicas y culturales de esa experiencia. 
Otro aspecto importante del significado, según este autor, es que el hecho de que algunas palabras denoten lo mismo va inmerso en los diferentes 'marcos' que las palabras puedan tener (Fillmore, 1982). Así, como ejemplo, plantea que la palabra 'tierra' no solo designa la parte seca de nuestro planeta, sino que denota la superficie seca en contraste con el mar: en el caso de la expresión 'tierra a la vista', que normalmente es emitida por los tripulantes de un barco, solo denota la superficie seca en contraste con el mar. Lo anterior permite concluir que existen diferentes 'marcos' para denotar el significado de la palabra 'tierra' y que este fenómeno sucede de la misma manera con el resto de las palabras.

Por otra parte, el Modelo Cognitivo Idealizado (MCI) engloba modelos complejos (convergencia de diferentes marcos) que combinan varios modelos individuales (Lakoff, 1987). El autor propone, por ejemplo, que en el caso de la palabra 'madre', el concepto está constituido por modelos cognitivos individuales que forman un solo modelo complejo Lakoff (1987: 74):

"MODELO NATAL: la madre es la persona que da a luz.

MODELO GENÉTICO: la madre es la mujer que contribuye con material genético.

MODELO CRIANZA: la madre es la persona femenina adulta que cría y educa al niño.

MODELO MARITAL: la madre es la esposa del padre.

MODELO GENEALÓGICO: la madre es el antepasado femenino más cercano".

De esta manera, el MCI de la palabra 'madre' comprende los cinco submodelos previamente descritos; es decir que, desde el punto de vista cognitivo, dicha palabra adquiere el valor de esas cinco acepciones. Ahora bien, hay otras expresiones compuestas con la palabra 'madre', como es el caso de 'madre adoptiva'. En este caso, el concepto se alejaría del MCI y formaría nuevos efectos prototípicos.

Por efecto prototípico se entiende la manera específica de comprender e interpretar una entidad y puede ser simple o complejo. El efecto prototípico simple es el que más se aproxima a la estructura de conocimiento generalizado comprendido por los hablantes y el complejo, el que requiere de nuevos elementos en la estructura del pensamiento para su comprensión; es decir, el que se aleja de su significado más elemental y gana matices semánticos diversos según los diferentes contextos en los que se presentan las palabras.

\section{Marco metodológico}

La ruta metodológica que se propuso en este trabajo se desarrolló en diferentes etapas. En primer lugar, se tomaron como base de trabajo 72 ejemplares electrónicos del periódico El Tiempo entre los años 2009-2010¹. En segundo lugar, se identificaron todos los neologismos formados por sufijación encontrados en dichos ejemplares y 
extraídos por la herramienta semiautomática BUSCANEO ${ }^{2}$ (Fase 1 para la construcción del corpus de análisis). En tercer lugar, con base en el criterio de frecuencia de aparición, se seleccionaron los neologismos formados con los sufijos ismo e -ista (Fase 2 para la construcción del corpus de análisis). En cuarto lugar, estos últimos se clasificaron en dos categorías: aquellos que tenían relación dual y aquellos que no la tenían (Muñoz, 2010). Por último, se analizaron los datos según los MCI y los efectos prototípicos simples y complejos.

\subsection{Corpus de análisis}

El corpus de análisis se consolidó en dos fases con los datos que reposan en la plataforma OBNEO y que forman parte del trabajo realizado por las Antenas Neológicas $^{3}$. La primera fase consistió en la selección de la totalidad de neologismos formados por sufijación en el corpus textual (137) con sus respectivas entradas (460). Es importante aclarar que la 'entrada' es el registro que se realiza en la plataforma OBNEO y que evidencia el neologismo y su contexto de uso, mientras que el 'neologismo' es la palabra nueva propiamente dicha (en este sentido, es posible que un mismo neologismo esté presente en diferentes entradas; esto es, en diferentes contextos). Para la segunda fase, se seleccionaron únicamente los neologismos con las formas léxicas -ismo e -ista puesto que presentaban una mayor frecuencia de aparición (60 neologismos en total con sus respectivas 204 entradas), siendo los más productivos en la variante del español de Colombia durante el período mencionado. Lo anterior puede evidenciarse en la Tabla 1:

Tabla 1. Construcción del corpus de análisis (Fases 1 y 2).

\begin{tabular}{|l|l|l|l|}
\hline \multicolumn{1}{|c|}{ Sufijos } & \multicolumn{1}{c|}{$\begin{array}{c}\text { Número de } \\
\text { neologismos }\end{array}$} & \multicolumn{1}{c|}{$\begin{array}{c}\text { Número de } \\
\text { entradas }\end{array}$} \\
\hline \multirow{5}{*}{ Fase 1 } & $\begin{array}{l}\text {-able, -aje, -al, -ano, -ante, -azgo, -ato, -ario, -azo, - } \\
\text { ción, -crón, -dad, -dor, -eo, -ear, -ente, -ense, } \\
\text {-eño, -ero, -esco, -í, -iano, -iar, -ico, -idad, -ismo, -ista, } \\
\text {-izar, -ivo, -itis, -ístico, -oso, -orio, -ora, } \\
\text {-miento, -menta, -tón, -ud. }\end{array}$ & 137 & 460 \\
\hline \multirow{2}{*}{ Fase 2 } & -ismo & 21 & 61 \\
\cline { 2 - 5 } & -ista & 39 & 143 \\
\cline { 2 - 5 } & Subtotal & 60 & 204 \\
\hline
\end{tabular}

Gracias al proceso de depuración del corpus de análisis en la segunda fase, logramos determinar la muestra final de los datos constituida por 60 neologismos formados con -ismo e -ista. Ver Tabla 2. 
Tabla 2. Neologismos formados por los sufijos -ismo e -ista 4 .

\begin{tabular}{|l|l|l|}
\hline Sufijos & $\begin{array}{l}\text { Número de } \\
\text { neologismos }\end{array}$ & Ejemplos \\
\hline -ismo & 21 & $\begin{array}{l}\text { agiotismo, antiuribismo, asistencialismo, bolivianarismo, chavismo, } \\
\text { ciclomontañismo, empresarismo, esclavismo, gobiernismo, guerrerismo, } \\
\text { harlismo, kartismo, kirchnerismo, menemismo, pandillismo, } \\
\text { paramilitarismo, pedalismo, peñalosismo, petrismo, soberanismo y } \\
\text { uribismo. }\end{array}$ \\
\hline -ista & 39 & $\begin{array}{l}\text { antichavista, asistencialista, basquetbolista, biogenetista, chavista, } \\
\text { clavadista, clientelista, colocolista, explosivista, facilista, forista, gaitanista, } \\
\text { guerrerista, hipnotista, inicialista, interista, interpartidista, kartista, } \\
\text { latinoamericanista, lefebvrista, menorrealista, mototaxista, pedalista, } \\
\text { pesista, recreacionista, reeleccionista, rescatista, revaluacionista, santista, } \\
\text { sonidista, supremacista, tallerista, tenimesista, tenismesista, terapista, } \\
\text { tiempista, uribista, voyerista y yihadista. }\end{array}$ \\
\hline
\end{tabular}

Una vez definido el corpus de análisis, los neologismos se organizaron sistemáticamente en dos categorías (Muñoz, 2010): según su relación dual; es decir, cuando estos dos sufijos compartían el mismo lexema; y según su relación no dual; es decir, cuando solo uno de estos utilizaba ya sea el sufijo -ismo o -ista. A continuación presentamos la Tabla 3 con algunos ejemplos que dan cuenta de la manera en que se estructuraron estas categorías: 
Tabla 3. Relación dual y no dual de los neologismos formados por los sufijos -ismo e -ista.

\begin{tabular}{|c|c|}
\hline RELACIÓN DUAL -ISMO / -ISTA & SIN RELACIÓN DUAL -ISMO / -ISTA \\
\hline $\begin{array}{l}\text { CHAVISMO - CHAVISTA } \\
\text { Pese a su deterioro, el colapso del 'chavismo' no está a } \\
\text { la vuelta de la esquina. } \\
\text { La politóloga Larissa Pizano dijo que las rabietas } \\
\text { 'chavistas' perjudican en Colombia a la Poloizquierda } \\
\text { pacifista. }\end{array}$ & $\begin{array}{l}\text { TENISMESISTA } \\
\text { La 'tenismesista', nacida en Cali y quien está } \\
\text { cerca de cumplir } 19 \text { años (el } 12 \text { de abril), es la } \\
\text { primera raqueta en la historia del país que } \\
\text { asistirá a las máximas justas del deporte. }\end{array}$ \\
\hline $\begin{array}{l}\text { PEDALISMO - PEDALISTA } \\
\text { El Tour de L'Avenir le puede significar al 'pedalismo' } \\
\text { nacional el regreso, el próximo año, a la élite del } \\
\text { ciclismo en Europa. } \\
\text { Los tres 'pedalistas' nacionales se enfrentarán al } \\
\text { venezolano José Rujano, actual campeón, y el español } \\
\text { Óscar Sevilla. }\end{array}$ & $\begin{array}{l}\text { FORISTA } \\
\text { Finalmente, hay intercambio de correos } \\
\text { electrónicos entre los dos 'foristas' para } \\
\text { finiquitar el encuentro. }\end{array}$ \\
\hline $\begin{array}{l}\text { ASISTENCIALISMO - ASISTENCIALISTA } \\
\text { El sistema pensional no es sostenible desde ningún } \\
\text { punto de vista con jubilaciones a edades tan } \\
\text { tempranas, el 'asistencialismo' no genera riqueza } \\
\text { alguna. } \\
\text { Los programas sociales de corte 'asistencialista' lo que } \\
\text { hacen es incrementar el círculo vicioso de la miseria. }\end{array}$ & $\begin{array}{l}\text { SOBERANISMO } \\
\text { El 'soberanismo' y la independencia han sido } \\
\text { utilizados por partidos catalanistas como CIU o } \\
\text { ERC, entre otros, para aprovechar el } \\
\text { descontento causado por la decisión del } \\
\text { Tribunal Constitucional. }\end{array}$ \\
\hline $\begin{array}{l}\text { GUERRERISMO - GUERRERISTA } \\
\text { El país nacional, hastiado del 'guerrerismo' del Estado, } \\
\text { anhela paz, debe rodear a la senadora y acompañarla } \\
\text { en su marcha resuelta tras este objetivo. } \\
\text { Asimismo, el senador indicó que la política } \\
\text { 'guerrerista', apoyada por Estados Unidos, ha llevado a } \\
\text { un desbordamiento de la guerra desde Colombia, } \\
\text { sobre las fronteras hacia Venezuela y hacia Ecuador. }\end{array}$ & $\begin{array}{l}\text { ESCLAVISMO } \\
\text { Me imagino los negros africanos que se } \\
\text { lucraban del 'esclavismo', tratando por todos } \\
\text { los medios de acallar las voces que denunciaban } \\
\text { este hecho. }\end{array}$ \\
\hline
\end{tabular}

\section{Análisis y resultados}

\subsection{Análisis semántico-cognitivo}

Una vez constituido el corpus de análisis, se tomaron los MCI a la luz de Lakoff (1987) en las dos categorías propuestas anteriormente. Este análisis se llevó a cabo con cada uno de los 61 neologismos formados por -ismo e -ista, a partir de dos aspectos inherentes a los MCI: en primer lugar, se tuvieron en cuenta los efectos prototípicos simples, los cuales permiten categorizar una palabra o conjunto de palabras dentro de una situación que se ajusta de manera perfecta al modelo (no entra en conflicto con el conocimiento que tiene el individuo acerca del concepto de la palabra); y en segundo lugar, se tomaron los efectos prototípicos complejos en los que se describe una falta de convergencia entre los distintos modelos (el concepto de la palabra varía según el contexto y el conocimiento del individuo) (Lakoff, 1987). Para efectos del presente artículo, presentaremos en detalle dos ejemplos del análisis que se llevó a cabo con dos neologismos que presentaban relación dual y dos que presentaban relación no dual. 
Con el fin de establecer relaciones semántico-cognitivas entre los neologismos formados por -ismo e -ista desde la Teoría de los MCI, estudiamos en detalle los contextos y realizamos diagramas que evidenciaban cómo los neologismos se enmarcaban en diferentes modelos. Así, por ejemplo, los neologismos 'kartismo' y 'pedalismo' tienen dos modelos: el deportivo y el profesional, los cuales están directamente relacionados con los efectos prototípicos simples. Esto es, que ambos neologismos pueden ser entendidos desde ambos modelos, según la situación comunicativa y el conocimiento del lector. Ver Figura 1.

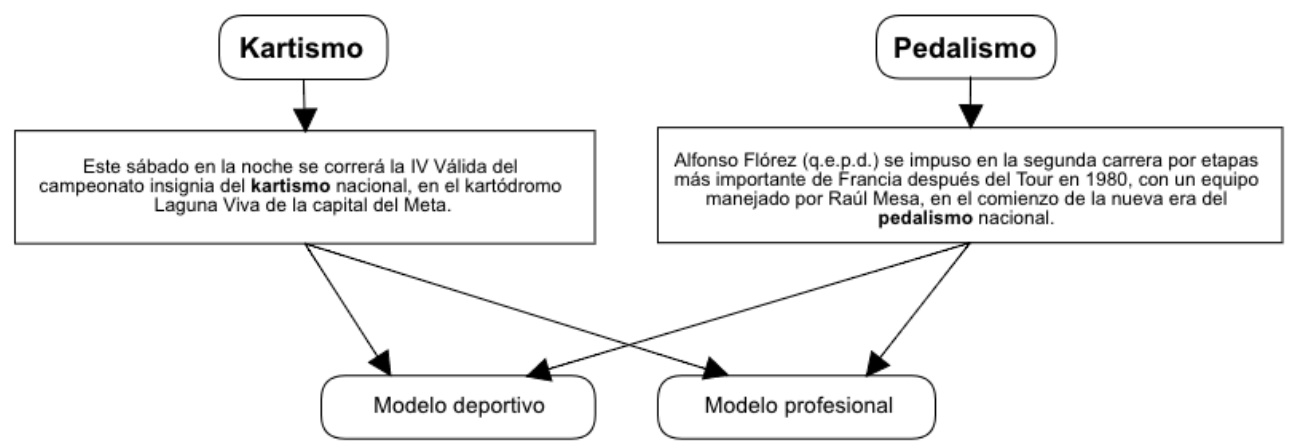

Figura 1. Ejemplo de las relaciones semántico-cognitivas de 'Kartismo’ y ‘Pedalismo’.

A continuación presentamos con mayor profundidad el análisis de algunos neologismos formados por los sufijos -ismo e -ista. En primera instancia, se muestran aquellos que tenían relación dual y sus efectos prototípicos simples y complejos desde la política y desde las prácticas deportivas y lúdicas y, en segunda instancia, se muestran aquellos que tenían una relación no dual y sus efectos prototípicos simples desde las prácticas deportivas, lúdicas y medioambientales. Estos temas emergieron de los datos analizados: los neologismos, en su gran mayoría, tenían una relación temática directa con ellos.

\subsection{Relación dual}

\subsubsection{Efectos prototípicos simples y complejos desde la política}

Como se mencionó en el marco teórico, los efectos prototípicos simples son los que más se acercan al pensamiento general de los hablantes; mientras que los efectos prototípicos complejos se alejan de la estructura del conocimiento general y, además, añaden otros elementos que facilitan su comprensión. En este caso, presentamos 'chavismo' y 'chavista' (neologismos con relación dual) como ejemplos claros en los cuales se evidencian los dos tipos de efectos prototípicos relacionados con diferentes modelos cognitivos. Tomamos en primer lugar el neologismo 'chavismo', como puede verse en las Figuras 2 y 3 : 


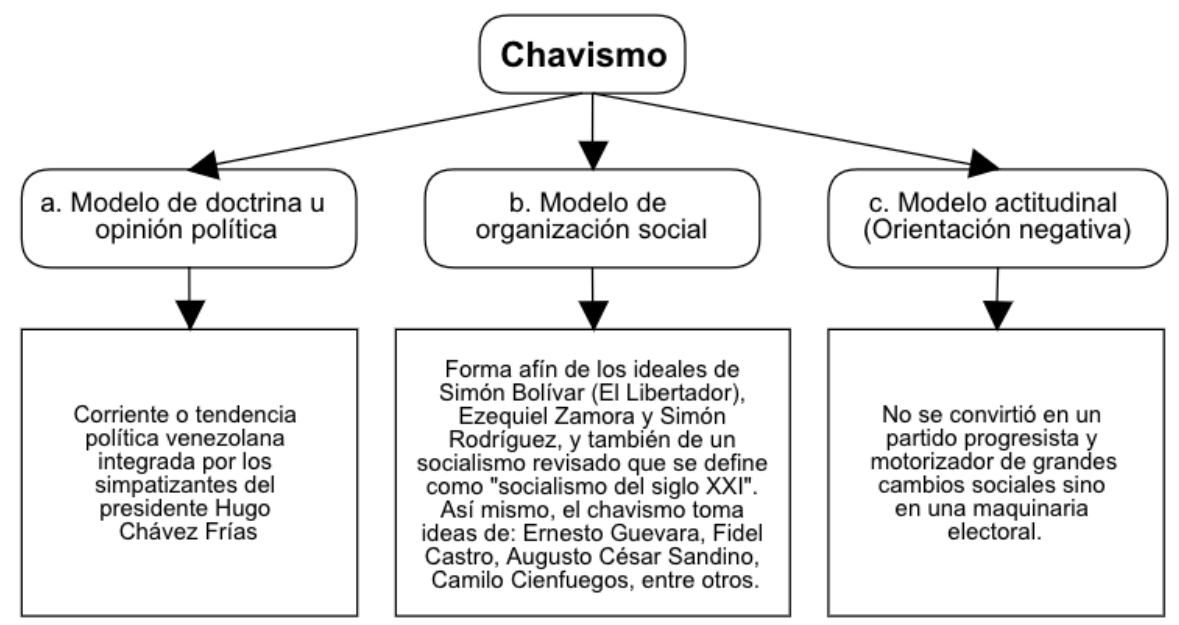

Figura 2. Ejemplo ‘Chavismo’.

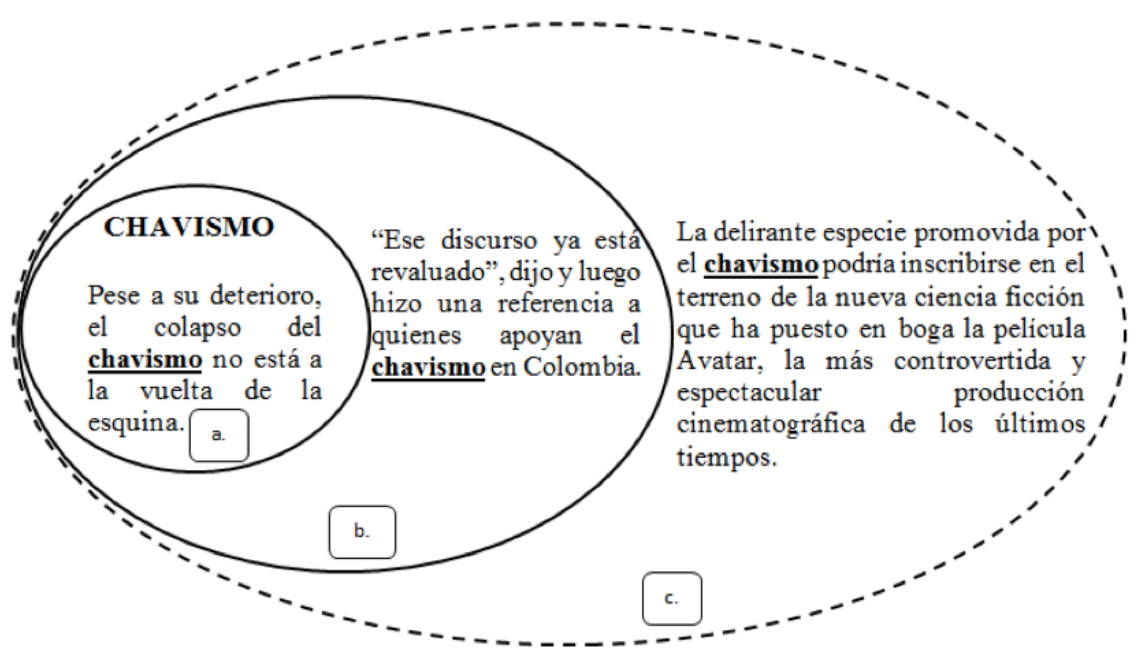

Figura 3. Ejemplos de efectos prototípicos para ‘Chavismo’ Modelos (a) y (b): Efectos prototípicos simples. Modelo (c): efectos prototípicos complejos.

En la Figura 2, el neologismo 'chavismo' se enmarca en tres modelos: (a) modelo de doctrina u opinión política, (b) modelo de organización social y (c) modelo actitudinal (orientación negativa). Estos modelos se corresponden con los contextos presentados en la Figura 3 y están enmarcados en la propuesta teórica de Lakoff (1987). En este sentido, los modelos (a) y (b) evidencian efectos prototípicos simples por cuanto se acercan a la realidad de los hablantes (en la prensa escrita), esto es, evocan al chavismo como una corriente o doctrina política (modelo (a)), así como una forma de organización social orientada al socialismo (modelo (b)). En el primer contexto, por ejemplo, hablar del colapso del chavismo puede entenderse como el 
deterioro de la tendencia política o corriente de los simpatizantes de Hugo Chávez (modelo (a)); mientras que en el segundo contexto, referirse al chavismo puede entenderse como la forma de apoyo de algunos sectores colombianos al movimiento socialista del siglo XXI con este nombre (modelo (b)).

Sin embargo, el modelo (c) evidencia un efecto prototípico complejo, puesto que se aleja del MCI de 'chavismo'. En este caso, hay nuevos elementos en el contexto presentado en la Figura 3 que nos permiten ver este fenómeno. Algunos de estos elementos son expresiones como 'delirante especie' y 'ciencia ficción', las cuales implican una connotación negativa que toma un grupo de personas caracterizadas por el despropósito, el disparate y lo irreal, que siguen una corriente política no como un partido progresista.

Como parte de la relación dual, presentamos en las Figuras 4 y 5 el análisis del neologismo 'chavista':

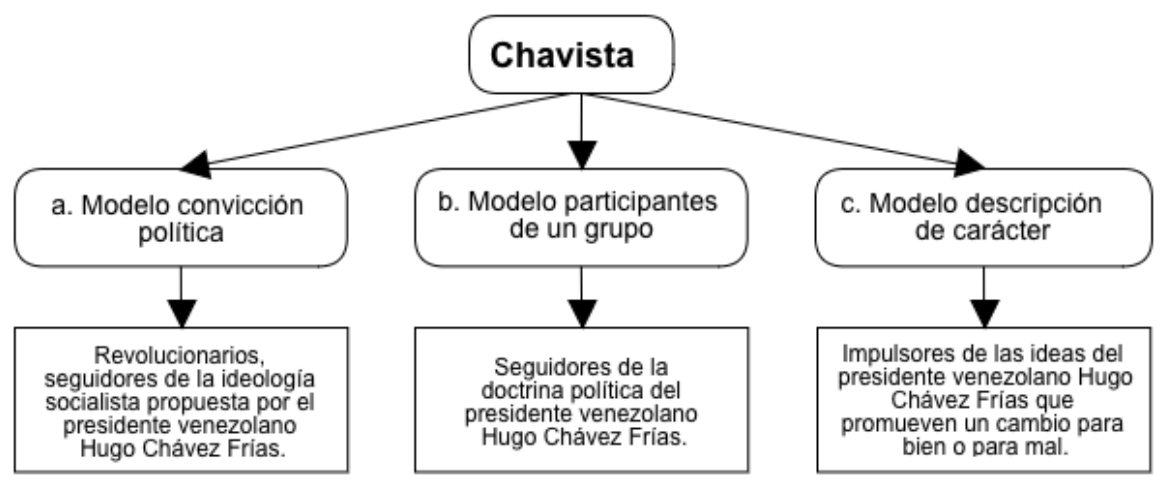

Figura 4. Ejemplo 'Chavista'.

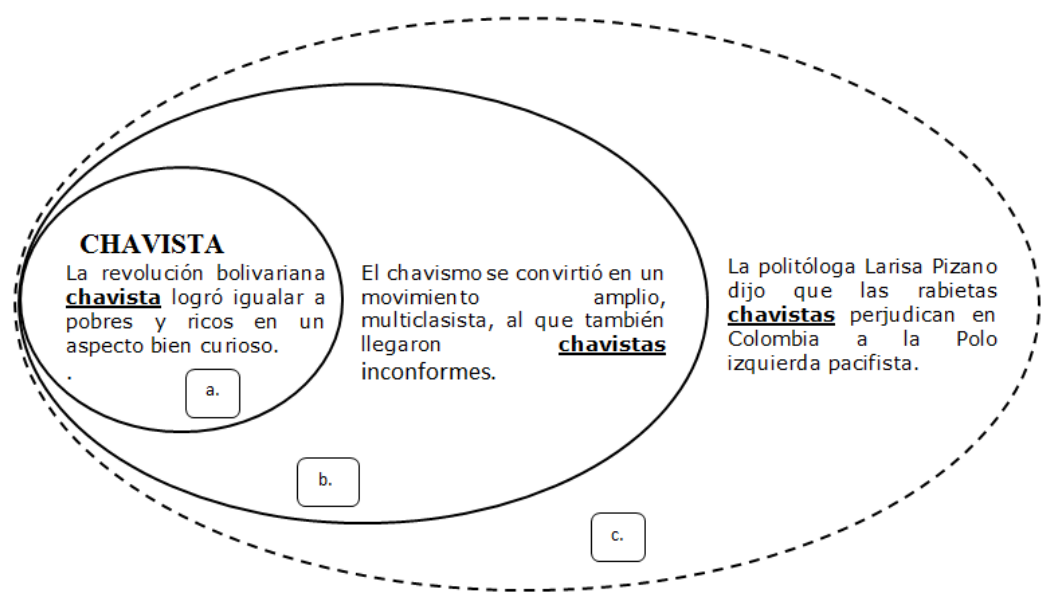

Figura 5. Ejemplos de efectos prototípicos para 'Chavista'Modelos (a) y (b): Efectos prototípicos simples. Modelo (c): Efectos prototípicos complejos. 
Al igual que en el caso de 'chavismo', la Figura 4 nos muestra que el neologismo 'chavista' se enmarca en tres modelos: (a) modelo convicción política, (b) modelo participantes de un grupo y (c) modelo descripción de carácter, a los cuales corresponden los contextos presentados en la Figura 5. De manera similar, los modelos (a) y (b) presentan efectos prototípicos simples porque tienen una relación directa con la estructura generalizada del pensamiento de los hablantes. Al mencionar la 'revolución bolivariana' en el primer contexto, entendemos 'chavista' como el revolucionario o seguidor de la ideología socialista de Hugo Chávez (modelo (a)); mientras que al hablar de un 'movimiento amplio' y 'multiclasista' en el segundo contexto, se hace mayor énfasis en el carácter de 'chavista' como una palabra que evoca al participante o al miembro de un grupo específico, que sigue dicha doctrina política (modelo (b)).

En contraste, el modelo (c) que está orientado hacia la descripción del carácter, evidencia un efecto prototípico complejo porque se aleja del MCI de 'chavista'. En este sentido, 'rabieta' es un elemento nuevo que se adhiere al neologismo 'chavista' formando una unidad sintagmática: 'rabieta chavista'. Esta nueva formación implica dos cambios; por un lado, la adición de un matiz semántico relacionado con el enfado, la impaciencia o el enojo (orientación negativa en la figura de Hugo Chávez); y, por otro lado, un cambio de categoría gramatical de 'chavista' como sustantivo a 'chavista' como adjetivo (para este caso en particular).

\subsubsection{Efectos prototípicos simples y complejos desde las prácticas deportivas}

Desde la temática del deporte, encontramos como ejemplos representativos de neologismos con relación dual a 'pedalismo' y 'pedalista'. En las Figuras 6 y 7 presentaremos el análisis del neologismo 'pedalismo':

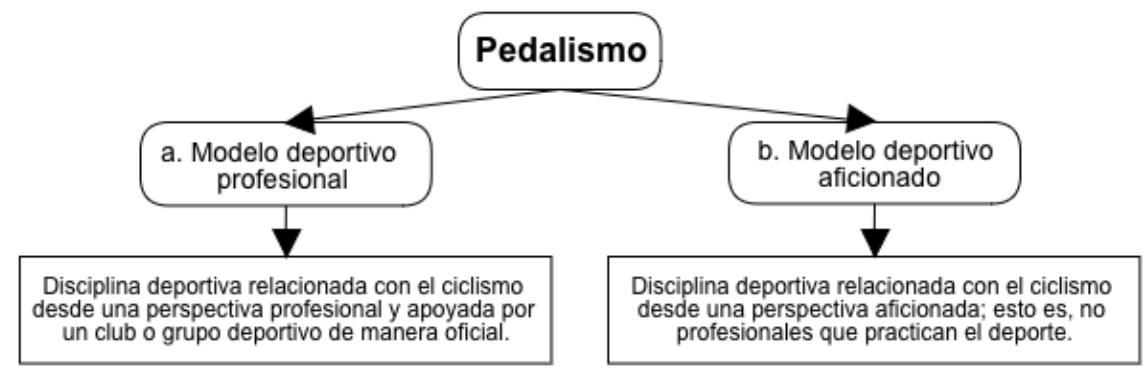

Figura 6. Ejemplo 'Pedalismo'. 


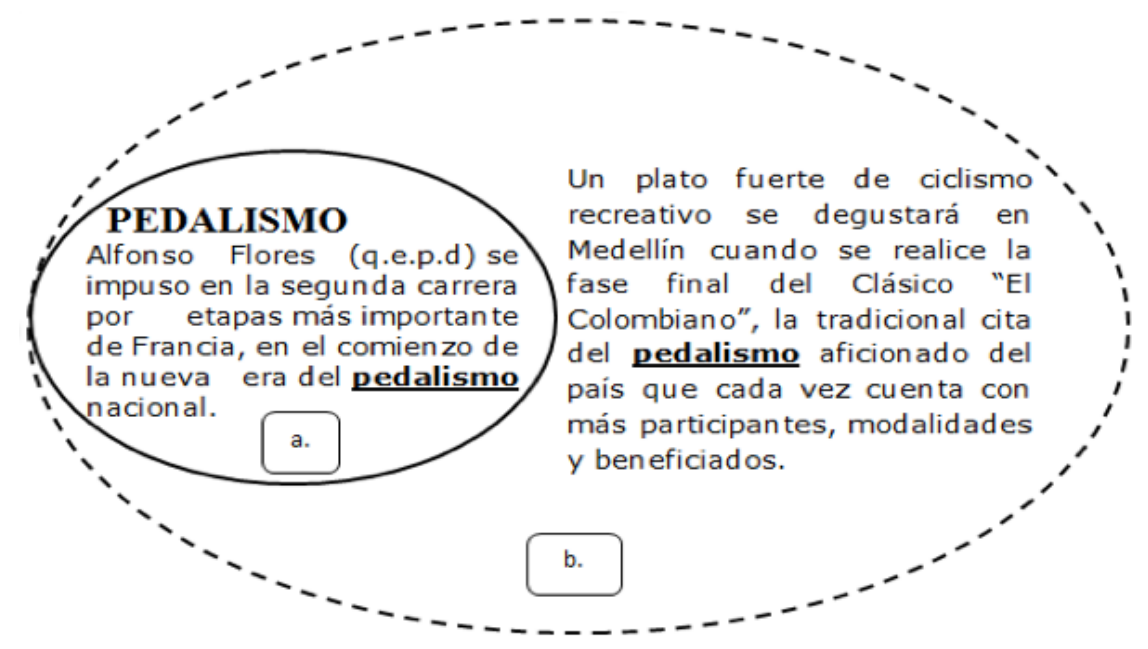

Figura 7. Ejemplos de efectos prototípicos para 'Pedalismo' Modelo (a): Efecto prototípico simple. Modelo (b): Efecto prototípico complejo.

Como lo ilustra la Figura 6, 'pedalismo' presenta dos modelos: (a) modelo deportivo profesional y (b) modelo deportivo aficionado. El MCI de este neologismo está más enfocado en su carácter profesional; esto quiere decir que la mayoría de contextos analizados desde la prensa escrita van dirigidos al ciclismo de élite en vueltas o tours nacionales o internacionales de alto reconocimiento. En el caso específico del primer contexto (modelo (a)), vemos que se genera un efecto prototípico simple puesto que concuerda con la definición anterior. Aspectos importantes como la 'segunda carrera', las 'etapas', 'Francia' (haciendo referencia al Tour de Francia) y, por último, la expresión sintagmática 'pedalismo nacional' evidencian que el neologismo se refiere a la disciplina deportiva profesional altamente reconocida.

En contraposición, el modelo (b) hace referencia al ciclismo desde una perspectiva aficionada. En la Figura 7, expresiones como 'ciclismo recreativo' y 'pedalismo aficionado' dejan entrever que, en este sentido, 'pedalismo' no se refiere de manera exclusiva al ciclismo profesional sino a la práctica libre, recreativa y lúdica del ciclismo por parte de las personas en general. Lo anterior hace que se genere un efecto prototípico complejo, puesto que se aleja del MCI de 'pedalismo' presentado anteriormente.

En las Figuras 8 y 9 se presenta el análisis del neologismo 'pedalista': 


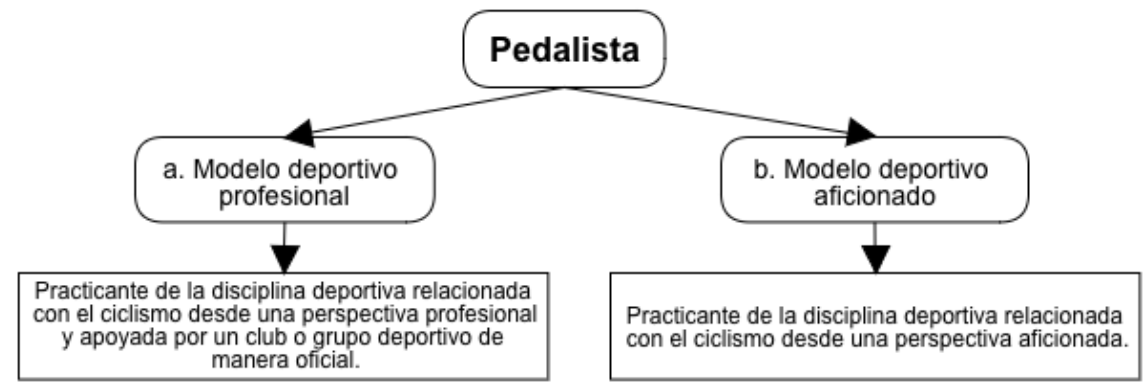

Figura 8. Ejemplo 'Pedalista'.

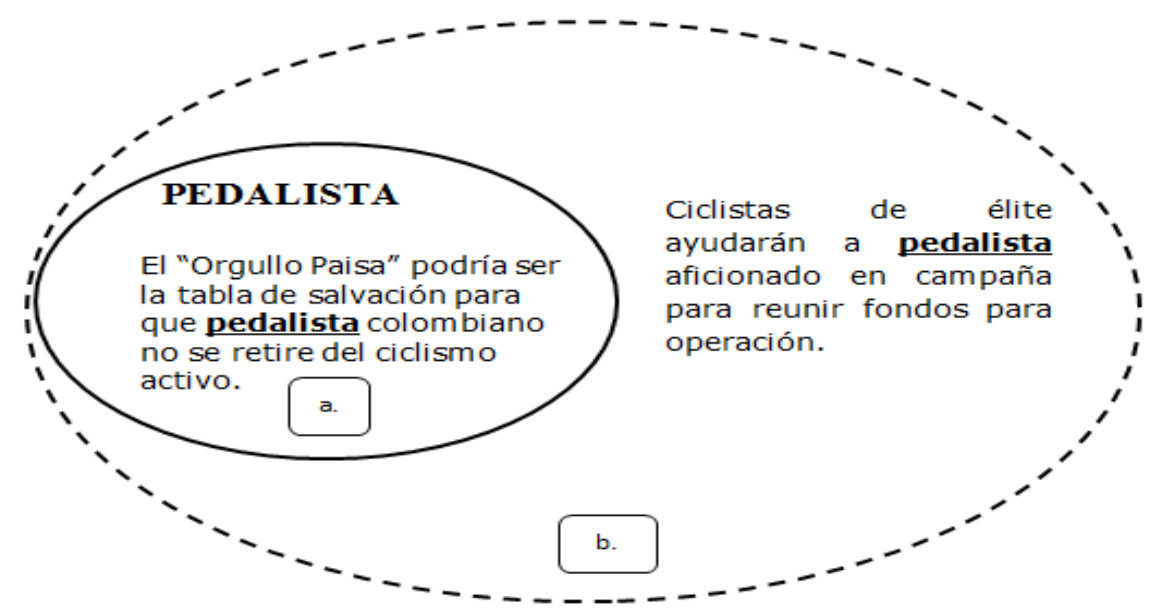

Figura 9. Ejemplos de efectos prototípicos para 'Pedalista’ Modelo (a): Efecto prototípico simple. Modelo (b): efecto prototípico complejo.

Como lo observamos en las Figuras 8 y 9, el neologismo 'pedalista' se enmarca en los mismos dos modelos de 'pedalismo' presentados anteriormente. Por una parte, en el caso particular del primer contexto (modelo (a)), expresiones como 'Orgullo Paisa' (relacionado con un equipo de ciclismo colombiano), 'tabla de salvación' y 'ciclismo activo' hacen pensar que se trata del profesional que practica el ciclismo. Por lo tanto, se genera un efecto prototípico simple en concordancia con lo que piensan los hablantes al usar el neologismo 'pedalista'.

Por otra parte, el segundo contexto (modelo (b)) se refiere a los practicantes no profesionales (pedalistas aficionados) de esta disciplina deportiva que serán ayudados por 'ciclistas de élite'. Esto constituye un efecto prototípico complejo. 


\subsection{Relación no dual}

\subsubsection{Efectos prototípicos simples desde las prácticas deportivas}

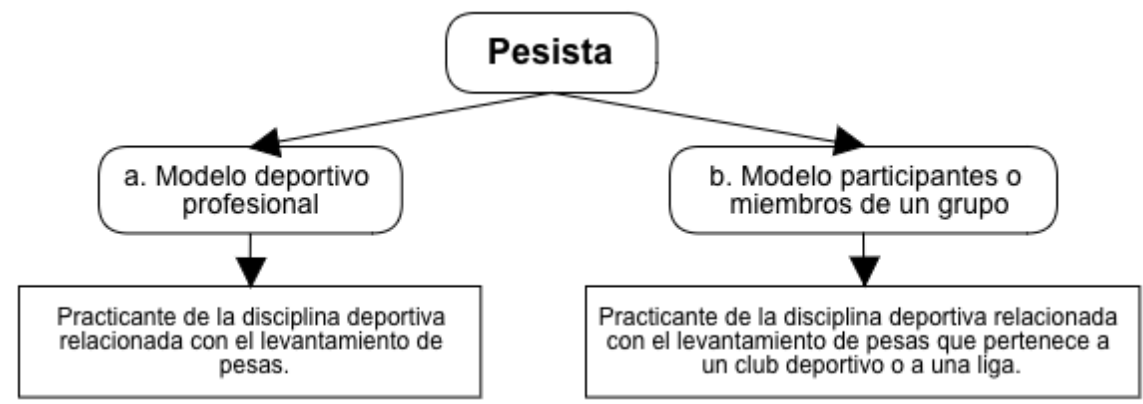

Figura 10. Ejemplo 'Pesista'.

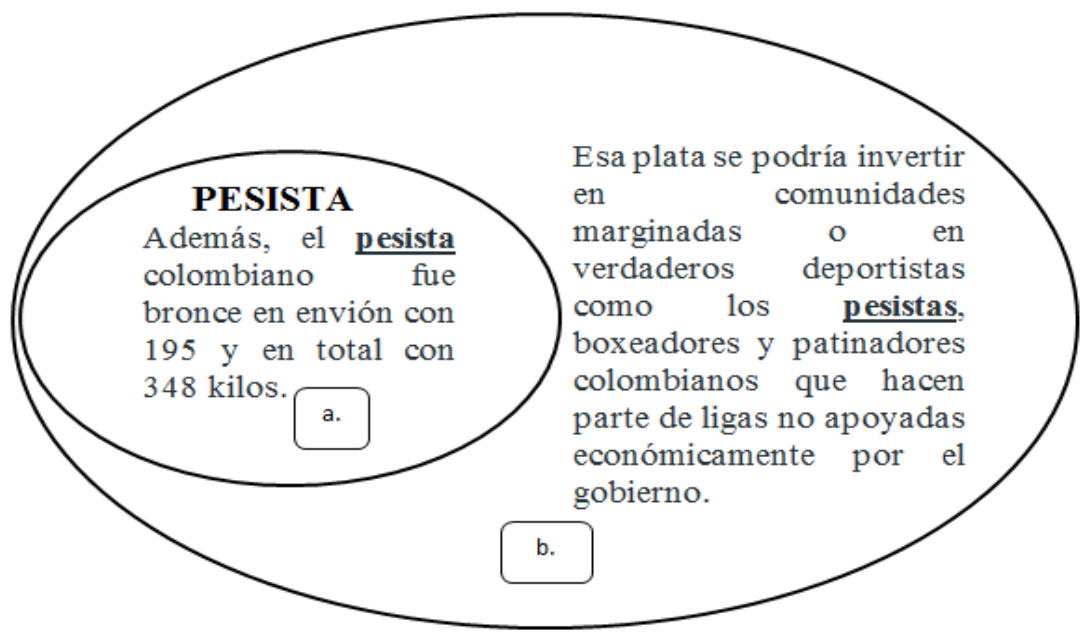

Figura 11. Ejemplo de efecto prototípico simple 'Pesista'.

Según las Figuras 10 y 11, el neologismo 'pesista' de relación no dual contempla dos modelos: (a) modelo deportivo profesional y (b) modelo participantes o miembros de un grupo. En ambos contextos, por ejemplo, el neologismo 'pesista' se refiere al practicante de la disciplina deportiva relacionada con el levantamiento de pesas (modelo (a)) y que a su vez hace parte de un grupo, club o liga deportiva (modelo (b)). 


\subsubsection{Efectos prototípicos simples desde el medio ambiente}

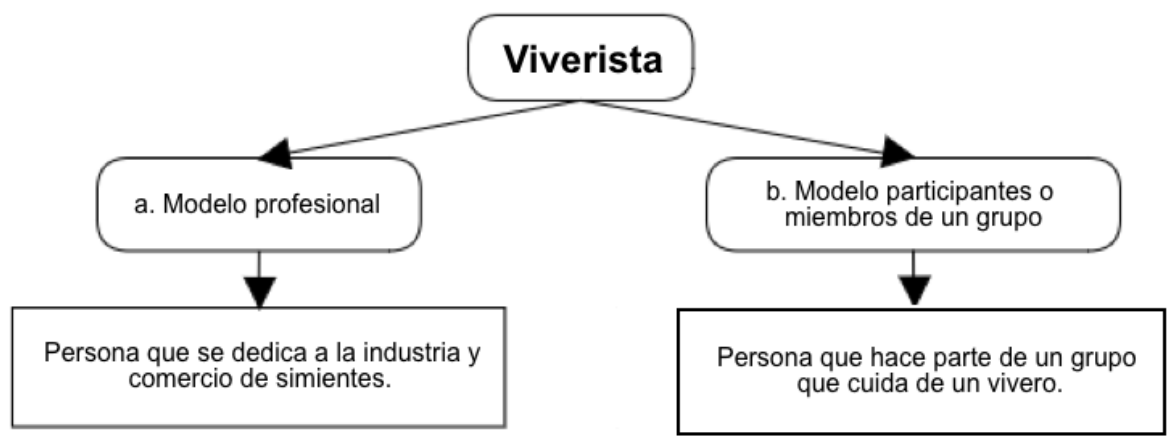

Figura 12. Ejemplo 'Viverista'.

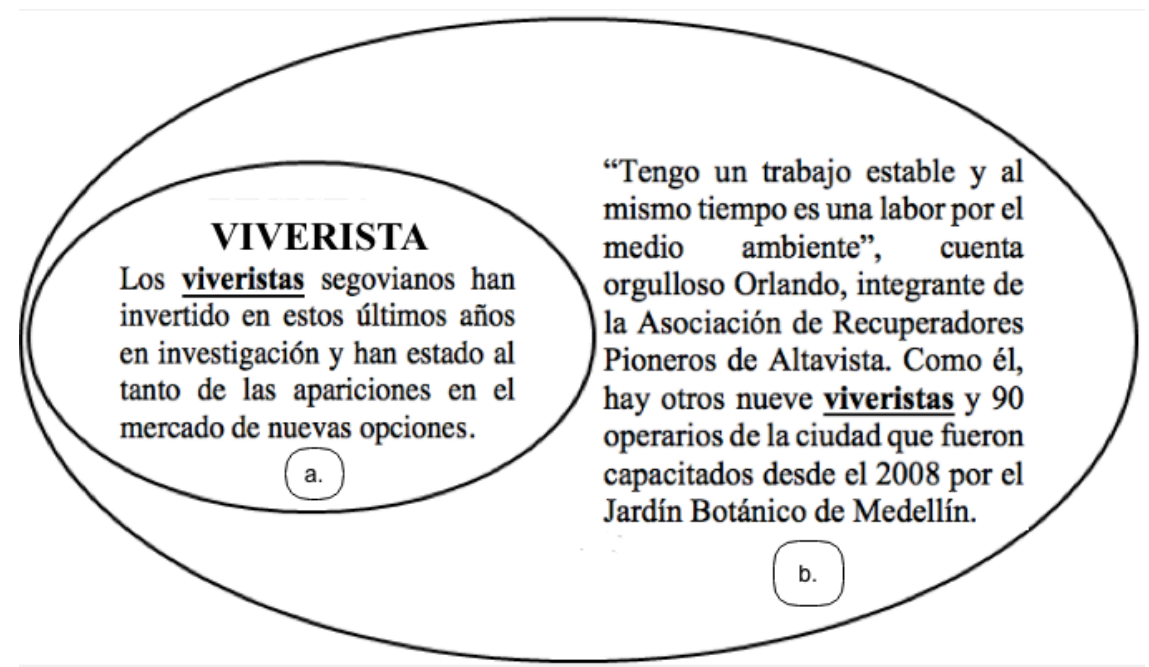

Figura 13. Ejemplo de efecto prototípico simple 'Viverista'.

De manera similar al neologismo 'pesista', como se presenta en las Figuras 12 y 13, el neologismo 'viverista' tiene dos modelos. El primero (a) está relacionado con la persona que se dedica profesionalmente a la industria, la producción, la comercialización y la investigación de semillas; mientras que el segundo (b) se refiere solo a la persona dedicada al cuidado de las semillas y que, a su vez, pertenece a un grupo dedicado a la misma tarea.

En este sentido, podemos evidenciar que, a diferencia de los neologismos que presentan relación dual, solamente se presentan efectos prototípicos simples para el caso de los neologismos de relación no dual. Esto podría obedecer a que las palabras evocan conceptos más precisos. 


\section{CONCLUSIONES}

A manera de conclusión, podemos decir que los neologismos formados con los sufijos -ismo e -ista han sido tradicionalmente analizados desde el punto de vista etimológico, morfosintáctico y semántico. En este trabajo se ha realizado un análisis desde la perspectiva semántico-cognitiva, que ha aportado elementos para comprender otros significados, especialmente, en lo relacionado con la manera de interpretar los neologismos objeto de estudio. Esto quiere decir que el presente trabajo permite la comprensión de unidades léxicas no solo desde lo literal, sino también desde los matices semántico-cognitivos que se evidencian en el uso de las palabras en diferentes contextos, con base en la Teoría de los Modelos Cognitivos Idealizados y la relación dual y no dual.

En primer lugar, encontramos que algunos de los neologismos formados por los sufijos que presentan correspondencia dual entre -ismo e -ista, por su carácter nominal, presentan una estructura semántico-cognitiva abstracta y están relacionados con Modelos Cognitivos Idealizados de doctrina o tendencia política y otros están asociados con Modelos Cognitivos Idealizados de disciplinas deportivas. En esta relación dual se encuentran algunos neologismos con efectos prototípicos simples y complejos: por un lado, al tratarse de efectos prototípicos simples, se evidencia cómo la palabra se acerca a la realidad generalizada de los hablantes que converge en un mismo Modelo Cognitivo Idealizado; por otro lado, al tratarse de efectos prototípicos complejos, se muestra cómo la palabra se aleja de dicha realidad generalizada de los hablantes, generando nuevos Modelos Cognitivos Idealizados.

En segundo lugar, los neologismos formados con el sufijo -ista, por su carácter adjetival, tienden a alejarse del Modelo Cognitivo Idealizado; es decir, a pesar de que la palabra es la misma, su significado gana otros matices semánticos según el contexto en el cual se presenta.

En tercer lugar, algunos neologismos formados con el sufijo -ista, que no tenían relación dual, están asociados con Modelos Cognitivos Idealizados de disciplinas deportivas y medioambientales. En esta relación no dual se evidencian solo efectos prototípicos simples, debido a que conducen a conceptos precisos y concretos, que se acercan al MCI.

En cuarto lugar, destacamos que la mayoría de los neologismos que no presentan correspondencia dual entre -ista e -ismo están formados con el sufijo -ista y están relacionados con disciplinas deportivas y medioambientales. Este fenómeno puede deberse al hecho de que ya existen en la lengua formal unidades léxicas aceptadas en el uso cotidiano, que no requieren de la adición del sufijo -ismo para lograr una nueva palabra. Algunos ejemplos de este fenómeno son: 'pesista' (levantamiento de pesas o halterofilia) y 'basquetbolista' (básquetbol o baloncesto). Por lo tanto, se hacen 
innecesarias las formaciones 'pesismo' o 'basquetbolismo' para completar la relación dual.

Por último, desde esta perspectiva semántico-cognitiva, hemos observado que los neologismos formados con el sufijo -ismo tienden a relacionarse más con conceptos abstractos como tendencias, movimientos y corrientes de diversa índole, que aquellos formados con el sufijo -ista. Lo anterior podemos relacionarlo con sus posibilidades de uso, articulación con el lenguaje y los discursos cotidianos.

\section{REFERENCIAS BIBLIOGRÁFICAS}

Alemany, J. (1920). Tratado de la formación de palabras en la lengua castellana. Madrid, Suárez.

Beniers, E. (1992). El sufijo -ismo en el español de México. En Scripta Philologica in honorem Juan M. Lope Blanch, II. Lingüistica Española e Iberoamericana. México: Universidad Nacional Autónoma de México.

Cabré, M. T. \& Rigau, G. (1986). Lexicologia i semàntica. Barcelona: Enciclopèdia Catalana.

Cabré, M. T., Bayà, R., Bernal, E., Freixa, J., Solé, E. \& Vallès, T. (2002). Evaluación de la vitalidad de una lengua a través de la neología: A propósito de la neología espontánea y de la neología planificada. En M.T. Cabré, J. Freixa, \& E. Solé, (Eds.), Lèxic i Neologia (pp. 159-201). Barcelona: Observatori de Neologia. Institut Universitari de Lingüística Aplicada, Universitat Pompeu Fabra.

Capanaga, P. (1999). Palabras de papel. Formaciones neológicas en español (19891999). Bolonia, CLUEB.

Capanaga, P. (2001). Productividad y Registro de las Formas Derivativas y Compositivas [en línea]. Disponible en: http://cvc.cervantes.es/literatura/aispi/pdf/14/14_101.pdf

Fillmore, C. (1975). An alternative to checklist theories of meaning. En C. Gogen, H. Thompson, G. Thurgood, K. Whistler \& J. Wright, (Eds.), Proceedings of the first annual meeting of the Berkeley Linguistic Society (pp.123-131). CA: Berkeley Linguistics Society.

Fillmore, C. (1977). Scenes-and-frames semantics. En A. Zampolli, (Ed.), Fundamental Studies in Computer Science (pp. 55-79). Ámsterdam: North Holland Publishing.

Fillmore, C. (1982). Frame semantics. En Linguistic Society of Korea (Ed.), Linguistics in the Morning Calm. Seúl, Hanshin Publishing Co.

Fillmore, C. (1985). Frames and semantics of understanding. Quaderni di semantica. 
Ibarretxe-Antuñano, I. \& Valenzuela, J. (2012). Lingüistica Cognitiva. Barcelona: Anthropos Editorial.

Lacuesta, R. S. \& Bustos, E. (1999). La derivación nominal. En I. Bosque \& V. Demonte, Gramática descriptiva de la lengua española (pp. 4505-4594). Madrid: Espasa Calpe.

Lakoff, G. (1983). Categories: An essay in cognitive linguistics. En Linguistic Society of Korea (Ed.), Linguistics in the Morning Calm. Seúl: Hanshin Publishing Co.

Lakoff, G. (1987). Women, Fire, and Dangerous Things: What Categories Reveal about the Mind. Chicago: The University of Chicago Press.

Lang, M. (1992). Formación de palabras en español. Morfología derivativa productiva en el léxico moderno. Madrid: Cátedra.

Meyer-Lübke, W. (1890-1906). Grammatik der romanischen Sprachen. París: Slatkine y Lafitte.

Muñoz, L. (2010) La historia de los derivados en -ismo e-ista en el español moderno. Tesis doctoral, Universidad Autónoma de Barcelona, Bellaterra, España [en línea]. Disponible en http://www.teseo.es.

Santana, O., Carrera, F., Pérez, J. \& Rodríguez, G. (2005). Una Aplicación para el Procesamiento de la Sufijación en Español. Grupo de Estructuras de Datos y Lingüistica Computacional. Universidad de las Palmas de Gran Canaria, España.

\section{NOTAS}

${ }^{1}$ Elegimos un período temporal específico (2009-2010) puesto que se trata de un estudio de tipo asincrónico. El Periódico El Tiempo es la fuente periodística de circulación nacional que tomamos como punto de referencia para este trabajo, debido a que es uno de los periódicos que procesa la Antena Neológica Colombiana.

2 BUSCANEO consiste en un extractor de neología formal que trabaja con textos de Internet. El programa está integrado a la plataforma OBNEO (Observatorio de Neología).

${ }^{3}$ El Proyecto Antenas Neológicas es liderado por el Observatorio de Neología (OBNEO) del Instituto Universitario de Lingüística Aplicada de la Universitat Pompeu Fabra de Barcelona (España) (wmm.iula.upf.edu/rec/antenas/index.htm). Es un proyecto conjunto para la observación y el estudio de la innovación léxica de las distintas variedades del español, del que forman parte los siguientes países: España, México, Colombia, Perú, Argentina y Chile.

${ }^{4}$ Con relación a estos ejemplos es importante aclarar que, al tratarse de un estudio de carácter asincrónico (2009-2010), se incluyeron datos que para esa fecha no aparecían registrados en el Diccionario de la Real Academia, ni en el Diccionario VOX Uso ni en el Diccionario de Colombianismos. 
${ }^{5}$ La palabra 'soberanista' no se considera un neologismo, puesto que ya aparece registrada en el Diccionario de la Real Academia de la Lengua Española.

${ }^{6}$ La palabra 'esclavista' no se considera un neologismo, puesto que ya aparece registrada en el Diccionario de la Real Academia de la Lengua Española. 